\title{
Serum homocysteine is associated with tubular interstitial lesions at the early stage of $\lg$ A nephropathy
}

\author{
Zizhen $\mathrm{Li}^{1 \dagger}$, Qianqian Han ${ }^{1 \dagger}$, Hongbo Ye ${ }^{1}$, Jiajia $\mathrm{Li}^{1}$, Xiaona Wei ${ }^{1}$, Rui Zhang ${ }^{1}$, Qiuyan Huang ${ }^{1}$, Yanchun Xu', \\ Guanxian Liu', Bin $\mathrm{Li}^{2^{*}}$ and Qiongqiong Yang ${ }^{1^{*}}$
}

\begin{abstract}
Background: The association between homocysteine (Hcy) and IgA nephropathy (IgAN) is not well understood. We aimed to investigate the relationship between Hcy and clinicopathologic features in IgAN patients.

Methods: A total of 337 IgAN patients and 150 sex- and age- matched healthy controls were enrolled in this singlecenter retrospective study. According to $\mathrm{Hcy} \leq 10 \mu \mathrm{mol} / \mathrm{L}$ or $>10 \mu \mathrm{mol} / \mathrm{L}$, patients were divided into low and high Hcy groups. Multivariate logistic regression was performed to explore the risk factors for elevated Hcy.

Results: Serum Hcy was higher in IgAN patients than in healthy controls $[11.6(9.1,15.3)$ vs. 8.8 $(7.5,10.6) \mu \mathrm{mol} / \mathrm{L}$, $P<0.001$ ], unanimously in the subgroup of 156 patients with a normal estimated glomerular filtration rate (eGFR) $(\geq$ $\left.90 \mathrm{ml} / \mathrm{min} / 1.73 \mathrm{~m}^{2}\right)[9.9(7.6,12.4)$ vs. $8.8(7.5,10.6) \mu \mathrm{mol} / \mathrm{L}, P<0.001]$. Compared to the low Hcy group, serum creatinine $(\mathrm{Scr})$, blood urine nitrogen (BUN), uric acid (UA), endocapillary hypercellularity (E) and tubular atrophy/interstitial fibrosis lesion (T) were higher in the high Hcy group. Hcy levels were positively correlated with Scr, BUN, UA, 24-h urine protein, and E and T lesions, but negatively correlated with eGFR and superoxide dismutase (SOD). In the subgroup with normal eGFR, patients with higher Hcy were persistent with higher Scr, BUN and T lesions. A multivariate logistic regression model showed that the risk of elevated Hcy in patients with pathological $T$ increased by 2.87 -fold. T lesions could better predict high Hcy, with an odds ratio (OR) of 14.20 in the subgroup with normal eGFR.
\end{abstract}

Conclusions: Pathologic T was an independent risk factor associated with elevated Hcy, especially at the early stage of IgAN.

Keywords: IgA nephropathy, Serum homocysteine, Tubular interstitial lesions

*Correspondence: neolibin@126.com; yangqq@mail.sysu.edu.cn

'Zizhen Li and Qianqian Han contributed equally to this work.

1 Department of Nephrology, Sun Yat-sen Memorial Hospital, Sun Yat-

sen University, No.107 Yanjiang West Road, Yuexiu District, Guangzhou, Guangdong, People's Republic of China

${ }^{2}$ Clinical Trials Unit, The First Affiliated Hospital, Sun Yat-sen University, No.58 Zhongshan 2nd Road, Yuexiu District, Guangzhou, Guangdong, People's Republic of China

\section{Background}

Homocysteine (Hcy) is a sulfhydryl amino acid situated at a branch point of methionine metabolism that is metabolized via two interrelated processes of remethylation and transsulfuration [1]. Numerous studies have found that elevated serum Hcy is a nontraditional independent risk factor for cardiovascular disease (CVD) [2, 3]. Elevated serum Hcy is a common finding in patients with chronic kidney disease (CKD) [4], which is attributed to reduced renal clearance of circulating Hcy and impaired Hcy metabolism [5]. Furthermore, elevated 
serum Hcy was confirmed to be related to the prevalence and progression of CKD $[6,7]$.

IgA nephropathy (IgAN) is the most common primary glomerulonephritis [8]. Approximately $30-40 \%$ of IgAN patients eventually develop end-stage renal disease (ESRD) within 20-30 years of renal biopsy [9]. A small retrospective study from Duan et al. revealed that elevated Hcy was associated with poor renal outcome in IgAN patients [10]. Another study by Mendelian randomization (MR) analysis observed positive effects of Hcy on serum creatinine, blood pressure (BP), and pathogenic $\mathrm{T}$ lesions in IgAN patients [11]. However, only the two small-sample studies mentioned above have investigated the relationship between Hcy and IgAN patients. Clinical research on the relationship between Hcy and pathology has not yet been highlighted.

In this study, we investigated the relationship between Hcy and clinicopathologic characteristics in IgAN patients in our center, especially at an early stage with a normal estimated glomerular filtration rate (eGFR) $(\geq$ $90 \mathrm{ml} / \mathrm{min} / 1.73 \mathrm{~m}^{2}$ ).

\section{Methods}

\section{Study design and participants}

This was a single-center retrospective study. Patients (age $\geq 14$ years) with biopsy-based diagnosis of primary IgAN between April 2014 and March 2021 in the Sun Yat-sen Memorial Hospital of Sun Yat-sen University were enrolled. Patients with secondary IgAN, such as systemic lupus erythematosus, Henoch-Schönlein purpura, anti-neutrophil cytoplasm antibody (ANCA)associated vasculitis, diabetic nephropathy or hepatic diseases were excluded from the study. Patients with fewer than 8 glomeruli in biopsy samples, incomplete data and CVDs such as stroke, coronary atherosclerotic heart disease, aortic aneurysm, and aortic dissection, were also excluded. Patients with Hcy $\leq 10 \mu \mathrm{mol} / \mathrm{L}$ and $\mathrm{Hcy}>10 \mu \mathrm{mol} / \mathrm{L}$ were classified into the low Hcy group and high Hcy group, respectively [11]. A total of 150 sexand age- matched healthy controls were recruited from the Center of Health Examination in order to explore whether serum Hcy was elevated in IgAN patients. This study was approved by the Medical Ethics Committee of Sun Yat-sen Memorial Hospital, Sun Yat-Sen University (approval number: No. SYSEC-KY-KS-2020-187), and exemption from informed consent of all patients was agreed upon.

\section{Demographic and clinicopathologic data collection}

Demographics and clinicopathologic data were retrieved from the medical record system. All laboratory indicators, including serum Hcy, were collected and measured at the time of biopsy and retrospectively analyzed.
Demographic data included sex, age, systolic blood pressure (SBP), diastolic blood pressure (DBP), mean arterial pressure (MAP) and history of hypertension (HBP). Laboratory indicators included 24-h urine protein, Hcy, serum creatinine ( $\mathrm{Scr}$ ), blood urine nitrogen (BUN), uric acid (UA), hemoglobin (Hb), serum albumin (ALB), fasting glucose (Glu), cholesterol (CHOL), triglyceride (TG), low-density lipoprotein-cholesterol (LDL), high-density lipoprotein-cholesterol (HDL), superoxide dismutase (SOD), high sensitivity $\mathrm{C}$ reaction protein (hsCRP), erythrocyte sedimentation rate (ESR), immunoglobulin A (IgA), immunoglobulin M (IgM), immunoglobulin G (IgG), complement 3 (C3), and complement 4 (C4). MAP was calculated as DBP plus a third of the pulse pressure. HBP was defined as $\mathrm{SBP} \geq 140 \mathrm{mmHg}$ and/ or $\mathrm{DBP} \geq 90 \mathrm{mmHg}$ or requirement for antihypertensive therapy. The eGFR was calculated using the Chronic Kidney Disease Epidemiology Collaboration (CKD-EPI) equation [12]. Blood samples were routinely collected in the morning after overnight fasting when hospitalized, and serum Hcy levels were measured using a Backman AU5831 automatic biochemistry analyzer (USA) before biopsy.

Renal biopsy specimens were examined by light, immunofluorescence, and electron microscopy. Two pathologists independently evaluated histopathological manifestations according to the Oxford classification system of IgAN [13]. Mesangial hypercellularity (M), endocapillary hypercellularity (E), segmental glomerulosclerosis $(\mathrm{S})$, tubular atrophy and interstitial fibrosis $(\mathrm{T})$ and crescents $(\mathrm{C})$ were recorded in detail.

\section{Statistical analysis}

Continuous variables with a normal distribution are expressed as the mean \pm standard deviation and were compared via Student's t test. Skewed distributed continuous variables were expressed as medians (interquartile ranges) and compared with the Mann-Whitney U test or the Kruskal-Wallis test. Categorical variables were expressed as frequencies (percentages) and compared via the chi-squared test. The $P$ value for multiple comparisons was corrected according to the Bonferroni method. Spearman's rank correlation was applied to detect the association between Hcy and clinicopathological features in IgAN patients. Univariate and multivariate logistic regression analyses were performed to identify the risk factors for elevated Hcy. A multivariate logistic regression model was created using stepwise forward LR to identify the variables that were independently associated with elevated Hcy selected from the variables with $P<0.1$ in univariate logistic regression analysis. We further carried out analysis in the subgroup with normal renal function patients based on eGFR $\geq 90 \mathrm{ml} / \mathrm{min} / 1.73 \mathrm{~m}^{2}$ 
to modify the effect of renal impairment. The data were analyzed using the SPSS 25.0 package (Chicago, IL, USA). All the $P$ values were two-tailed, and $P<0.05$ was considered significant in all statistical tests.

\section{Results}

\section{Serum Hcy and clinicopathologic parameters of IgAN} patients

A total of 337 IgAN patients aged $34.0(27.5,44.0)$ years entered the study, and $40.1 \%(n=135)$ were male. The age of the healthy controls was $36.0(30.0,40.0)$ years, and $42.7 \%(n=64)$ were male. The median levels of eGFR and MAP were $88.1(62.8,103.4) \mathrm{ml} / \mathrm{min} / 1.73 \mathrm{~m}^{2}$ and 94.7 $(88.7,106.3) \mathrm{mmHg}$, respectively. The median level of 24-h urine protein was $0.6(0.2,1.7) \mathrm{g} / 24 \mathrm{~h}$.

Serum levels of Hcy $[11.6(9.1,15.3)$ vs. $8.8(7.5,10.6)$ $\mu \mathrm{mol} / \mathrm{L}, P<0.001$; Fig. 1] were significantly higher in the IgAN group than in the healthy control group. A total of 156 patients had normal renal function with eGFR

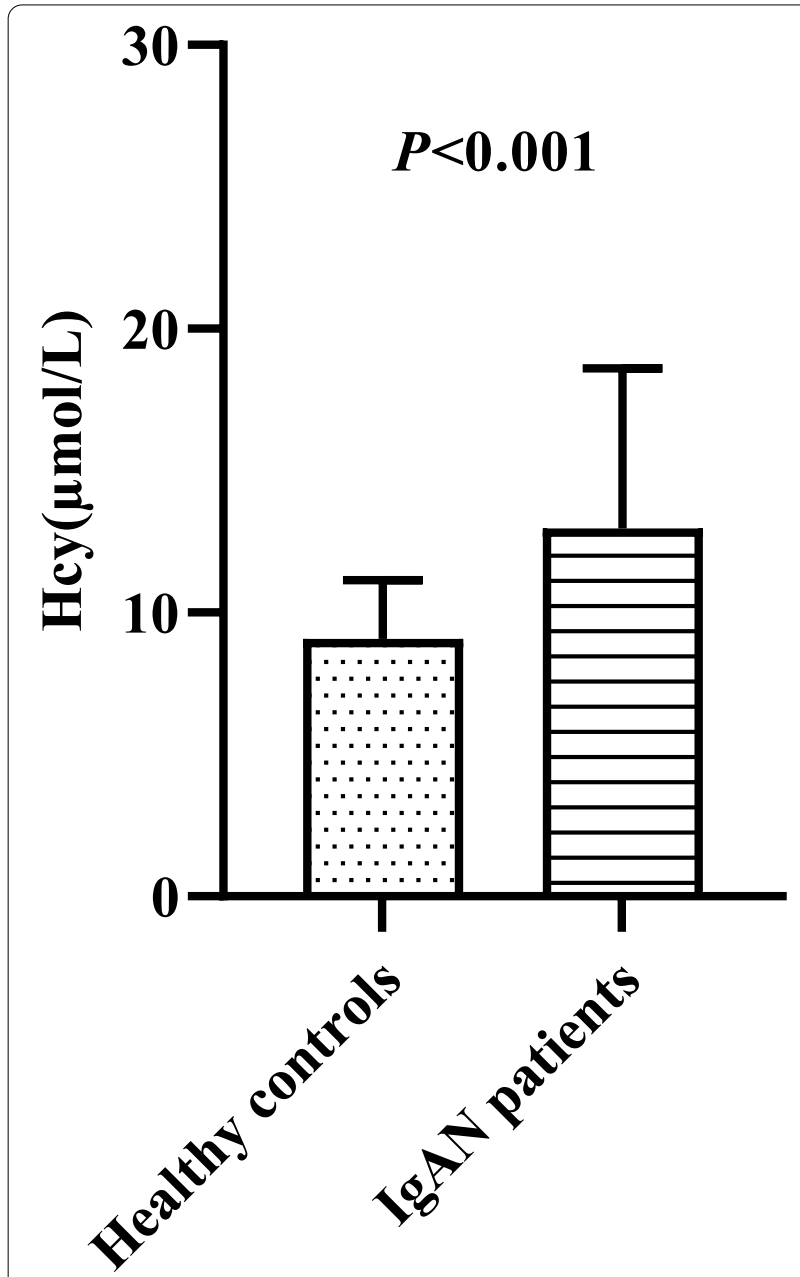

Fig. 1 Comparison of serum Hcy between healthy controls and IgAN patients $\geq 90 \mathrm{ml} / \mathrm{min} / 1.73 \mathrm{~m}^{2}$, and 74 patients were in the high Hcy group. The median level of Hcy was 9.9 (7.6, 12.4) $\mu \mathrm{mol} / \mathrm{L}$ in CKD stage 1 patients, which was still higher than that in healthy controls $(P<0.001)$. The median Hcy levesl of CKD stage 1-5 patients were 9.9 (7.6, 12.4), 11.1 (9.6, 13.7), 15.7 (12.9, 18.8), 24.4 (16.1, 27.0), and 23.8 $(16.9,31.7) \mu \mathrm{mol} / \mathrm{L}$, respectively $(P<0.001$; Fig. 2$)$. Hcy was $13.5(10.5,17.5) \mu \mathrm{mol} / \mathrm{L}$ and $10.7(8.1,13.6) \mu \mathrm{mol} / \mathrm{L}$ in male and female patients $(P<0.001)$, respectively. Compared with patients without endocapillary hypercellularity (E0), those with endocapillary hypercellularity (E1) had significantly elevated Hcy $[13.2(10.4,15.8)$ vs. $11.2(8.9,14.9) \mu \mathrm{mol} / \mathrm{L}, P=0.027$; Fig. 3]. Patients with more severe tubular atrophy/interstitial fibrosis lesions showed significantly higher serum levels of Hcy $\left[\mathrm{T}_{2}: 22.7\right.$ $(15.7,30.0)$ vs. $\mathrm{T}_{1}: 13.6(11.2,15.7)$ vs. $\mathrm{T}_{0}: 10.5(8.3,13.4)$ $\mu \mathrm{mol} / \mathrm{L}, P<0.001$; Fig. 3].

\section{Comparison of clinicopathologic characteristics} between the low Hcy group and the high Hcy group A total of 114 patients were in the low Hcy group and 223 patients were in the high Hcy group. The clinical characteristics and histopathological features are presented in Table 1. Compared with the low Hcy group, patients in the high Hcy group were older $(P=0.003)$, had a higher proportion of male patients $(P<0.001)$ and had a history of HBP $(P<0.001)$. Significantly higher levels of MAP $(P<0.001)$, Scr $(P<0.001)$, BUN $(P<0.001)$,

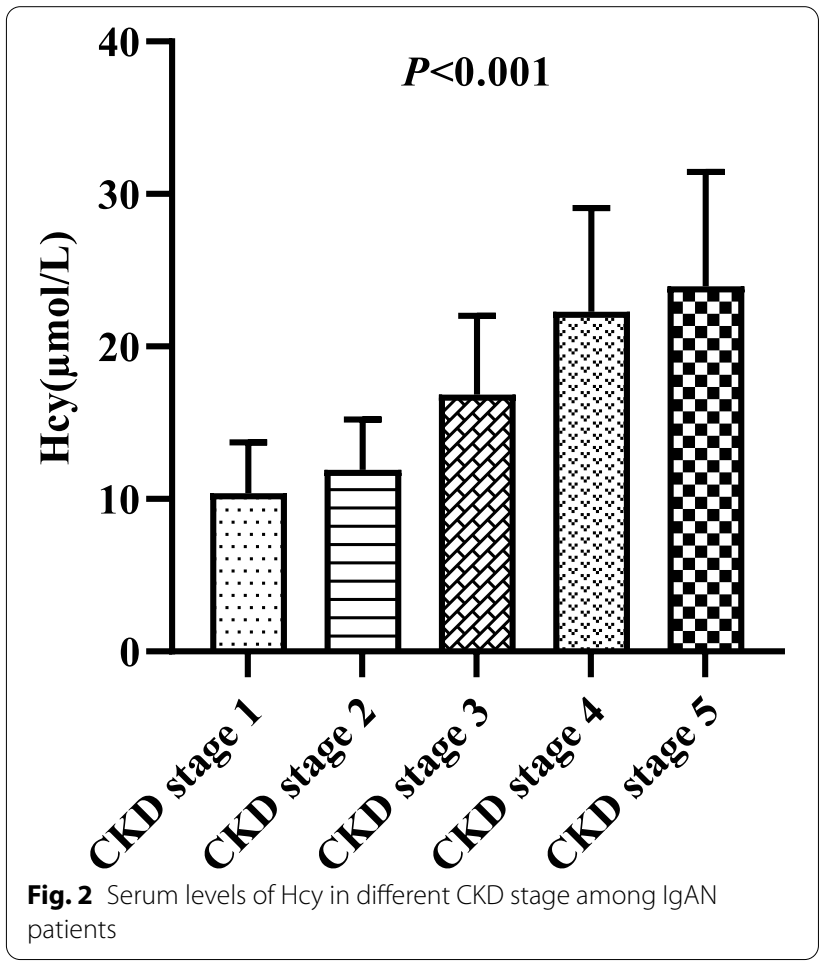




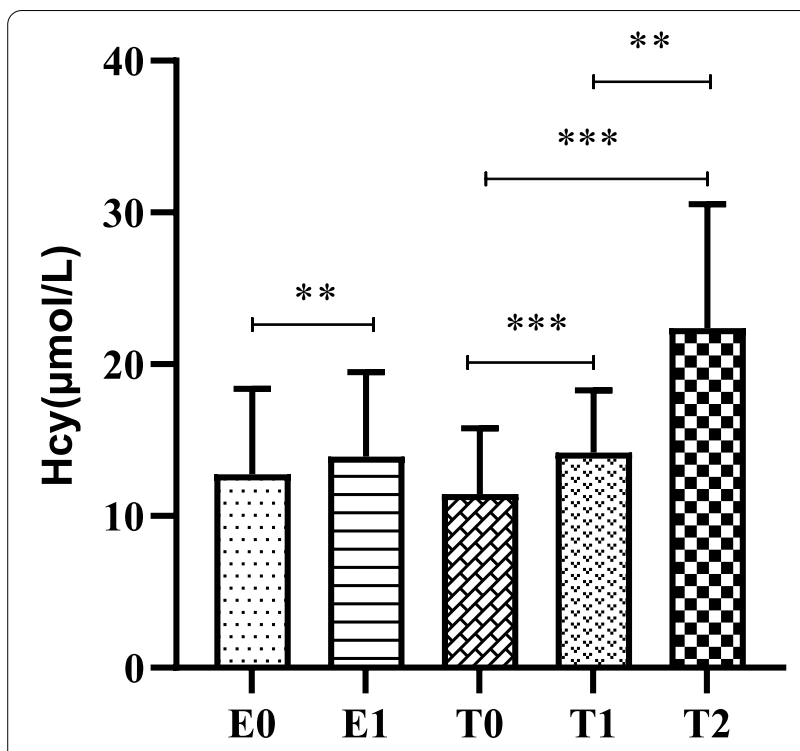

Pathologic lession

Fig. 3 Comparison of serum Hcy in lesions of endocapillary hypercellularity (E) and tubular atrophy/interstitial fibrosis $(T)$ among IgAN patients. ${ }^{* *} P<0.05,{ }^{* * *} P<0.001$

UA $(P<0.001)$, ALB $(P=0.011)$, and hsCRP $(P=0.033)$ and lower levels of eGFR $(P<0.001)$, HDL $(P<0.001)$, and IgM $(P=0.024)$ were shown in the high Hcy group. Pathological E $(P=0.041)$ and $\mathrm{T}(P<0.001)$ lesions were significantly more serious in the elevated Hcy group than in the low Hcy group. Difference in 24-h urine protein, $\mathrm{Hb}$ and complement were not discovered between the two groups.

\section{Correlations between Hcy and clinicopathologic parameters}

Hcy levels were positively correlated with age $(r=0.18$, $P=0.001)$, MAP $(r=0.24, \quad P<0.001), \quad$ Scr $\quad(r=0.69$, $P<0.001)$, BUN $(r=0.57, \quad P<0.001), \quad$ UA $\quad(r=0.42$, $P<0.001), 24-$ h urine protein $(r=0.22, P<0.001)$, TG $(r=0.21, P<0.001)$, hsCRP $(r=0.22, P<0.001)$, ESR $(r=0.13, \quad P=0.016), \quad C 4 \quad(r=0.14, P=0.010)$, pathologic E $(r=0.12, P=0.026)$, and pathologic $\mathrm{T}(r=0.45$, $P<0.001)$, but negatively correlated with eGFR $(r=-0.62, P<0.001), \mathrm{Hb}(r=-0.15, P=0.008)$, HDL $(r=-0.31, P<0.001)$, SOD $(r=-0.23, P<0.001)$, and IgM $(r=-0.16, \quad P=0.004)$. However, relationships between Hcy and ALB, Glu, CHOL, LDL, IgA, IgG, C3, and pathologic $\mathrm{M}, \mathrm{S}$, and $\mathrm{C}$ were not found $(P>0.05)$. The correlations between Hcy and the clinicopathological features of IgAN patients are shown in Table 2.
Risk factor analysis of IgAN associated with elevated Hcy The logistic regression analysis results are shown in Table 3. Univariate analysis revealed that IgAN patients who had lower levels of HDL and IgM, and higher levels of age, MAP, Scr, BUN, UA, ALB, ESR, and pathologic $E$ and $T$ lesions were at a greater risk of elevated Hcy. After the variable selection procedure, the final multivariate logistic regression model showed that male sex [odds ratio $(\mathrm{OR})=3.47,95 \%$ confidence interval $(\mathrm{CI})=1.86-6.48, P<0.001)$ ], lower eGFR $(\mathrm{OR}=0.95,95 \%$ $\mathrm{CI}=0.94-0.97, P<0.001)$, higher $\mathrm{ALB}(\mathrm{OR}=1.07,95 \%$ $\mathrm{CI}=1.03-1.12, P=0.001)$, and pathologic $\mathrm{T}(\mathrm{OR}=2.87$, 95\% $\mathrm{CI}=1.20-6.89, P=0.018)$ independently increased the risk of elevated Hcy in IgAN patients.

\section{Subgroup analysis in IgAN patients with normal renal function}

A total of 156 patients had normal renal function with eGFR $\geq 90 \mathrm{ml} / \mathrm{min} / 1.73 \mathrm{~m}^{2}$. Seventy-four patients were in the high Hcy group, with a higher proportion of male patients, and a history of HBP, SBP, Scr, BUN, ALB, and pathological $\mathrm{T}$ lesions and a lower eGFR and 24-h urine protein than those in the low Hcy group. The results of the subgroup analysis are presented in Supplement Table 1. As shown in Supplement Table 2, Hcy levels were positively correlated with Scr, BUN, UA, ALB and $\mathrm{T}$ lesions, but negatively correlated with eGFR and HDL. Moreover, multivariate logistic regression analysis adjusted by male sex, eGFR, and ALB also showed that pathologic $\mathrm{T}$ was more related to elevated Hcy $(\mathrm{OR}=14.20,95 \% \mathrm{CI}=1.68-120.03, P=0.015)$ in the subgroup of patients with $\mathrm{eGFR} \geq 90 \mathrm{ml} / \mathrm{min} / 1.73 \mathrm{~m}^{2}$.

\section{Discussion}

In this study, we found that the serum Hcy of IgAN patients was significantly higher than that of healthy controls in our center. IgAN patients with elevated Hcy displayed more severe $\mathrm{T}$ lesions, even in subgroups of patients with normal renal function. Multivariate logistic regression analysis showed that pathologic $\mathrm{T}$ was an independent risk factor for elevated Hcy in IgAN patients and persisted in a subgroup analysis according to eGFR $\geq 90 \mathrm{ml} / \mathrm{min} / 1.73 \mathrm{~m}^{2}$.

In this study, we found that Hcy was positively correlated with age in the whole cohort of 337 IgAN patients. A serious shortage of vitamin B12 and vitamin B6 was common in elderly populations, and a lack of cofactors reduced the metabolism of Hcy. In addition, the decline in renal function with aging also caused elevation of Hcy [14]. Significantly higher serum levels of Hcy were observed in male patients with IgAN than that in female patients $[13.5(10.5,17.5)$ vs. 10.7 (8.1, 
Table 1 Clinicopathologic characteristics of IgAN patients in low and high Hcy group

\begin{tabular}{|c|c|c|c|c|}
\hline Variable & Total $(n=337)$ & $\mathrm{Hcy} \leq 10.0(n=114)$ & Hcy $>10.0(n=223)$ & $P$ \\
\hline $\operatorname{Hcy}(\mu \mathrm{mol} / \mathrm{L})$ & $11.6(9.1,15.3)$ & $8.2(7.3,9.1)$ & $13.7(11.7,16.7)$ & $<0.001^{* *}$ \\
\hline Age (years) & $34.0(27.5,44.0)$ & $32.5(27.0,39.0)$ & $35.0(28.0,47.0)$ & $0.003^{*}$ \\
\hline Male, n (\%) & $135(40.1)$ & $27(23.7)$ & $108(48.4)$ & $<0.001^{* *}$ \\
\hline $\mathrm{SBP}(\mathrm{mmHg})$ & $123.0(115.0,137.5)$ & $119.5(110.0,131.0)$ & $126.0(117.0,141.0)$ & $<0.001^{* * *}$ \\
\hline $\mathrm{DBP}(\mathrm{mmHg})$ & $80.0(74.0,90.0)$ & $77.5(73.0,87.0)$ & $82.0(75.0,94.0)$ & $0.002^{*}$ \\
\hline MAP $(\mathrm{mmHg})$ & $94.7(88.7,106.3)$ & $92.0(86.3,99.7)$ & $96.3(89.3,109.7)$ & $<0.001^{* *}$ \\
\hline HBP, n (\%) & $132(39.2)$ & $22(19.3)$ & $110(49.3)$ & $<0.001^{* *}$ \\
\hline $\operatorname{Scr}(\mu \mathrm{mol} / \mathrm{L})$ & $86.0(70.0,115.5)$ & $69.5(61.0,82.3)$ & $99.0(77.0,139.0)$ & $<0.001^{* *}$ \\
\hline $\operatorname{eGFR}\left(\mathrm{mL} / \mathrm{min} / 1.73 \mathrm{~m}^{2}\right)$ & $88.1(62.8,103.4)$ & $101.4(89.3,115.4)$ & $75.0(44.9,95.2)$ & $<0.001^{* *}$ \\
\hline CKD stage, $n(\%)$ & & & & $<0.001^{* *}$ \\
\hline CKD 1 & $156(46.3)$ & $82(71.9)$ & $74(33.2)$ & \\
\hline CKD 2 & $100(29.7)$ & $32(28.1)$ & $68(30.5)$ & \\
\hline CKD 3 & $51(15.1)$ & $0(0.0)$ & $51(22.9)$ & \\
\hline CKD 4 & $13(3.9)$ & $0(0.0)$ & $13(5.8)$ & \\
\hline CKD 5 & $17(5.0)$ & $0(0.0)$ & $17(7.6)$ & \\
\hline BUN (mmol/L) & $5.1(4.3,6.9)$ & $4.5(3.6,5.1)$ & $5.8(4.7,8.0)$ & $<0.001^{* *}$ \\
\hline UA (mmol/L) & $390.0(324.0,484.5)$ & $352.5(302.8,413.8)$ & $413.0(347.0,512.0)$ & $<0.001^{* *}$ \\
\hline 24-h urine protein ( $\mathrm{g} / 24 \mathrm{~h}$ ) & $0.6(0.2,1.7)$ & $0.5(0.2,1.0)$ & $0.7(0.3,1.8)$ & 0.101 \\
\hline $\mathrm{Hb}(\mathrm{g} / \mathrm{L})$ & $125.0(114.0,139.5)$ & $128.5(118.8,138.0)$ & $124.0(111.0,142.0)$ & 0.135 \\
\hline$A L B(g / L)$ & $35.0 \pm 6.8$ & $33.7 \pm 7.9$ & $35.6 \pm 6.2$ & $0.011^{*}$ \\
\hline Glu (mmol/L) & $4.4 \pm 0.6$ & $4.4 \pm 0.5$ & $4.4 \pm 0.6$ & 0.631 \\
\hline $\mathrm{CHOL}(\mathrm{mmol} / \mathrm{L})$ & $5.1(4.3,5.9)$ & $5.1(4.5,6.0)$ & $5.0(4.3,5.9)$ & 0.262 \\
\hline $\mathrm{TG}(\mathrm{mmol} / \mathrm{L})$ & $1.6 \pm 1.4$ & $1.4 \pm 0.9$ & $1.7 \pm 1.6$ & 0.080 \\
\hline LDL (mmol/L) & $3.2(2.6,3.8)$ & $3.2(2.7,3.9)$ & $3.2(2.6,3.8)$ & 0.386 \\
\hline $\mathrm{HDL}(\mathrm{mmol} / \mathrm{L})$ & $1.3 \pm 0.4$ & $1.4 \pm 0.4$ & $1.2 \pm 0.3$ & $<0.001^{* *}$ \\
\hline $\mathrm{SOD}(\mathrm{U} / \mathrm{ml})$ & $136.0 \pm 26.6$ & $138.4 \pm 30.3$ & $134.8 \pm 24.5$ & 0.241 \\
\hline $\mathrm{hsCRP}(\mathrm{mg} / \mathrm{L})$ & $0.7(0.3,1.8)$ & $0.5(0.3,1.5)$ & $0.8(0.3,2.0)$ & $0.033^{*}$ \\
\hline $\mathrm{ESR}(\mathrm{mm} / \mathrm{h})$ & $17.0(9.0,32.0)$ & $16.5(10.0,27.0)$ & $19.0(8.0,35.0)$ & 0.647 \\
\hline $\lg A(g / L)$ & $3.3 \pm 1.1$ & $3.3 \pm 1.1$ & $3.3 \pm 1.2$ & 0.695 \\
\hline $\lg M(g / L)$ & $1.2 \pm 0.6$ & $1.3 \pm 0.6$ & $1.2 \pm 0.5$ & $0.024^{*}$ \\
\hline $\lg G(g / L)$ & $11.5 \pm 3.3$ & $11.0 \pm 3.5$ & $11.7 \pm 3.2$ & 0.066 \\
\hline C3 (mg/L) & $1054.2 \pm 202.2$ & $1077.9 \pm 213.5$ & $1042.0 \pm 195.7$ & 0.125 \\
\hline C4 (mg/L) & $258.4 \pm 91.6$ & $250.9 \pm 91.1$ & $262.2 \pm 91.9$ & 0.288 \\
\hline \multicolumn{5}{|l|}{ Oxford classification, n (\%) } \\
\hline M1 & $320(95.0)$ & $108(94.7)$ & $212(95.1)$ & 0.896 \\
\hline E1 & $65(19.3)$ & $15(13.2)$ & $50(22.4)$ & $0.041^{*}$ \\
\hline S1 & $132(39.2)$ & $42(36.8)$ & $90(40.4)$ & 0.531 \\
\hline $\mathrm{T}$ & & & & $<0.001^{* * *}$ \\
\hline T0 & $238(70.6)$ & $106(93.0)$ & $132(59.2)$ & \\
\hline $\mathrm{T} 1$ & 70 (20.8) & $7(6.1)$ & $63(28.3)$ & \\
\hline $\mathrm{T} 2$ & $29(8.6)$ & $1(0.9)$ & $28(12.6)$ & \\
\hline C & & & & 0.524 \\
\hline $\mathrm{CO}$ & $150(44.5)$ & $54(47.4)$ & $96(43.0)$ & \\
\hline C1 & $166(49.3)$ & $55(48.2)$ & $111(49.8)$ & \\
\hline$C 2$ & $21(6.2)$ & $5(4.4)$ & $16(7.2)$ & \\
\hline
\end{tabular}

Abbreviations: Hcy homocysteine, SBP systolic blood pressure, DBP diastolic blood pressure, MAP mean arterial pressure, $H B P$ Hypertension, $S c r$ serum creatinine, eGFR estimated glomerular filtration rate, $C K D$ chronic kidney disease, $B U N$ blood urine nitrogen, $U A$ uric acid, $H b$ hemoglobin, $A L B$ serum albumin, Glu fasting glucose, $C H O L$ cholesterol, $T G$ triglyceride, $L D L$ low-density lipoprotein-cholesterol, $H D L$ high-density lipoprotein-cholesterol, SOD superoxide dismutase, $h s C R P$ high sensitivity $C$ reaction protein, $E S R$ erythrocyte sedimentatio rate, $I g A$ immunoglobulin $\mathrm{A}$, IgM immunoglobulin $\mathrm{M}$, IgG immunoglobulin $\mathrm{G}, \mathrm{C} 3$ complement3, $C 4$ complement4, $M$ mesangial hypercellularity, $E$ endocapillary hypercellularity, $S$ segmental glomerulosclerosis, $T$ tubular atrophy and interstitial fibrosis, $C$ crescents

${ }^{*} P<0.05$, ${ }^{* *} P<0.001$ 
Table 2 Correlations between Hcy and clinicopathologic parameters in IgAN patients

\begin{tabular}{|c|c|c|}
\hline Variable & $r$ & $P$ \\
\hline Age & 0.18 & $0.001^{*}$ \\
\hline MAP & 0.24 & $<0.001^{* *}$ \\
\hline Scr & 0.69 & $<0.001^{* *}$ \\
\hline eGFR & -0.62 & $<0.001^{* *}$ \\
\hline BUN & 0.57 & $<0.001^{* *}$ \\
\hline UA & 0.42 & $<0.001^{* *}$ \\
\hline 24-h urine protein & 0.22 & $<0.001^{* *}$ \\
\hline $\mathrm{Hb}$ & -0.15 & $0.008^{*}$ \\
\hline ALB & 0.05 & 0.338 \\
\hline Glu & 0.02 & 0.695 \\
\hline $\mathrm{CHOL}$ & -0.02 & 0.718 \\
\hline TG & 0.21 & $<0.001^{* *}$ \\
\hline LDL & 0.01 & 0.834 \\
\hline $\mathrm{HDL}$ & -0.31 & $<0.001^{* *}$ \\
\hline SOD & -0.23 & $<0.001^{* *}$ \\
\hline hsCRP & 0.22 & $<0.001^{* *}$ \\
\hline ESR & 0.13 & $0.016^{*}$ \\
\hline $\lg A$ & 0.04 & 0.435 \\
\hline $\lg M$ & -0.16 & $0.004^{*}$ \\
\hline $\lg G$ & 0.07 & 0.214 \\
\hline C3 & 0.03 & 0.533 \\
\hline C4 & 0.14 & $0.010^{*}$ \\
\hline M & 0.01 & 0.869 \\
\hline$E$ & 0.12 & $0.026^{*}$ \\
\hline S & 0.04 & 0.500 \\
\hline $\mathrm{T}$ & 0.45 & $<0.001^{* *}$ \\
\hline C & -0.01 & 0.822 \\
\hline
\end{tabular}

Abbreviations: MAP mean arterial pressure, Scr serum creatinine, eGFR estimated glomerular filtration rate, $B U N$ blood urine nitrogen, $U A$ uric acid, $\mathrm{Hb}$ hemoglobin, $A L B$ serum albumin, Glu fasting glucose, $\mathrm{CHOL}$ cholesterol, $T G$ triglyceride, $L D L$ low-density lipoprotein-cholesterol, $H D L$ high-density lipoprotein-cholesterol, SOD superoxide dismutase, hSCRP high sensitivity $C$ reaction protein, ESR erythrocyte sedimentatio rate, IgA immunoglobulin A, IgM immunoglobulin M, IgG immunoglobulin G, C3 complement3, C4 complement4, $M$ mesangial hypercellularity, $E$ endocapillary hypercellularity, $S$ segmental glomerulosclerosis, $T$ tubular atrophy and interstitial fibrosis, $C$ crescents ${ }^{*} P<0.05,{ }^{* *} P<0.001$

13.6) $\mu \mathrm{mol} / \mathrm{L}, P<0.001]$, which was similar to a previous study $[15,16]$. Moreover, males were independently associated with elevated Hcy in our study. One possible reason was the difference between the sexes in lifestyle variables that are known to have an impact on Hcy [17]. In addition, estrogen could decrease plasma Hcy levels [18]. As previously reported [19], patients with elevated Hcy had higher ALB levels. The mechanism was not well elucidated. Scholars have explained that Hcy covalently binds to ALB, resulting in the lack of filtration and absorption processes of Hcy in the kidney [20].
However, more studies are needed to probe the link between ALB and Hcy and its underlying mechanism.

From the results in this study, it can be seen that serum Hcy was closely related to kidney function. A previous study demonstrated that serum Hcy was very likely elevated in patients with CKD and gradually increased with the progression of CKD stage [4]. Hcy was markedly elevated at the ESRD stage [21], and was 3-5 times that of healthy participants [22]. In our study, the serum Hcy of IgAN patients was similarly elevated compared to that of sex- and age- matched healthy controls, even in a subgroup of patients with normal renal function, and increased with the decline in eGFR. However, there was a question as to whether the elevated Hcy was a cause for CKD or rather the result of CKD.

It has been demonstrated that the kidney is a major site for the removal of plasma Hcy and plays an important role in the regulation of plasma Hcy levels [23]. In our study, multivariate logistic regression analysis found that pathologic T $(\mathrm{OR}=2.87,95 \% \mathrm{CI}=1.20-6.89, P=0.018)$ was an independent risk factor for elevated Hcy in IgAN patients, even after adjustment for eGFR and in the subgroup with normal renal function $(\mathrm{OR}=14.20$, 95\% $\mathrm{CI}=1.68-120.03, P=0.015)$. Zhang et al. observed the association between Hcy and pathologic $\mathrm{T}$ lesions in IgAN [11]. Given impairment in remethylation, patients with CKD were likely more dependent on the pathway of Hcy transsulfuration, which was catalyzed by cystathione- $\beta$-synthase (CBS) and $\gamma$-cystathionase (CTL). Meanwhile, James D et al. expressed that CBS was enriched in the proximal convoluted tubule cells. CTLs exhibit higher enrichment patterns in proximal straight tubule cells [24]. Li et al. found that renal proximal tubules were major sites for Hcy metabolism in the kidney [25]. When tubular atrophy and interstitial fibrosis occurr, the transsulfuration pathway is reduced, leading to the elevation of Hcy. It seemed that the increase in Hcy was the result of renal injury, especially $\mathrm{T}$ lesions.

The Oxford classification score is a well-regarded prognostic indicator for IgAN. Tubular interstitial lesions are the most valuable histological parameter and a final pathway for most progressive kidney diseases. T lesions are common in IgAN patients even with normal renal function; however, factors associated with $\mathrm{T}$ lesions in IgAN have not been elucidated. Possible mechanisms to explain the $\mathrm{T}$ lesions are that with aggravating renal impairment, renal units will be damaged accompanied by fibrosis, resulting in loss of normal excretory function and reduced clearance of Hcy. On the other hand, IgAN is an inflammatory disease, and Hcy is related to the inflammatory response and stimulates the production of cytokines and proinflammatory molecules, which may be related to renal fibrosis. We found that nearly half 
Table 3 Clinicopathologic features as factors of elevated Hcy by univariate and multivariate logistic regression analysis

\begin{tabular}{|c|c|c|c|c|c|c|}
\hline \multirow[t]{3}{*}{ Variables } & \multirow{2}{*}{\multicolumn{2}{|c|}{ Univariate }} & \multicolumn{4}{|l|}{ Multivariate } \\
\hline & & & \multicolumn{2}{|l|}{ Overall } & \multicolumn{2}{|c|}{$\mathrm{eGFR} \geq 90 \mathrm{ml} / \mathrm{min} / 1.73 \mathrm{~m}^{2}$} \\
\hline & Odds ratio $(95 \% \mathrm{Cl})$ & $P$ & Odds ratio $(95 \% \mathrm{Cl})$ & $P$ & Odds ratio $(95 \% \mathrm{Cl})$ & $P$ \\
\hline Age & $1.04(1.02-1.06)$ & $<0.001^{* *}$ & & & & \\
\hline Male & $3.03(1.83-5.02)$ & $<0.001^{* *}$ & $3.47(1.86-6.48)$ & $<0.001^{* *}$ & $5.31(2.23-12.64)$ & $<0.001^{* *}$ \\
\hline MAP & $1.04(1.02-1.05)$ & $<0.001^{* *}$ & & & & \\
\hline Scr & $1.07(1.05-1.09)$ & $<0.001^{* *}$ & & & & \\
\hline eGFR & $0.95(0.94-0.96)$ & $<0.001^{* *}$ & $0.95(0.94-0.97)$ & $<0.001^{* *}$ & $0.95(0.91-0.98)$ & $0.003^{*}$ \\
\hline BUN & $1.66(1.40-1.98)$ & $<0.001^{* *}$ & & & & \\
\hline UA & $1.01(1.00-1.01)$ & $<0.001^{* *}$ & & & & \\
\hline 24-h urine protein & $1.00(0.94-1.08)$ & 0.929 & & & & \\
\hline $\mathrm{Hb}$ & $0.99(0.98-1.00)$ & 0.085 & & & & \\
\hline ALB & $1.04(1.01-1.08)$ & $0.013^{*}$ & $1.07(1.03-1.12)$ & $0.001^{*}$ & $1.10(1.04-1.16)$ & $0.001^{*}$ \\
\hline Glu & $0.91(0.60-1.36)$ & 0.630 & & & & \\
\hline $\mathrm{CHOL}$ & $0.87(0.77-1.00)$ & $0.042^{*}$ & & & & \\
\hline TG & $1.24(0.98-1.58)$ & 0.079 & & & & \\
\hline LDL & $0.83(0.69-1.01)$ & 0.061 & & & & \\
\hline HDL & $0.31(0.16-0.61)$ & $0.001^{*}$ & & & & \\
\hline SOD & $1.00(0.99-1.00)$ & 0.241 & & & & \\
\hline hsCRP & $1.01(0.98-1.04)$ & 0.429 & & & & \\
\hline ESR & $1.01(1.00-1.02)$ & $0.033^{*}$ & & & & \\
\hline $\lg A$ & $1.04(0.85-1.28)$ & 0.694 & & & & \\
\hline $\lg M$ & $0.63(0.42-0.95)$ & $0.027^{*}$ & & & & \\
\hline $\lg G$ & $1.07(1.00-1.14)$ & 0.068 & & & & \\
\hline C3 & $1.00(1.00-1.00)$ & 0.126 & & & & \\
\hline C4 & $1.00(1.00-1.00)$ & 0.288 & & & & \\
\hline MO & 1 (reference) & & & & & \\
\hline M1 & $1.07(0.39-2.97)$ & 0.896 & & & & \\
\hline E0 & 1 (reference) & & & & & \\
\hline E1 & $1.91(1.02-3.57)$ & $0.044^{*}$ & & & & \\
\hline SO & 1 (reference) & & & & & \\
\hline S1 & $1.16(0.73-1.85)$ & 0.532 & & & & \\
\hline T0 & 1 (reference) & & 1 (reference) & & 1 (reference) & \\
\hline $\mathrm{T} 1-2$ & $9.13(4.24-19.66)$ & $<0.001^{* *}$ & $2.87(1.20-6.89)$ & $0.018^{*}$ & $14.20(1.68-120.03)$ & $0.015^{*}$ \\
\hline $\mathrm{CO}$ & 1 (reference) & & & & & \\
\hline C1-2 & $1.19(0.76-1.87)$ & 0.451 & & & & \\
\hline
\end{tabular}

Abbreviations: MAP mean arterial pressure, Scr serum creatinine, eGFR estimated glomerular filtration rate, $B U N$ blood urine nitrogen, UA uric acid, $H b$ hemoglobin, $A L B$ serum albumin, Glu fasting glucose, $C H O L$ cholesterol, $T G$ triglyceride, $L D L$ low-density lipoprotein-cholesterol, $H D L$ high-density lipoprotein-cholesterol, $S O D$ superoxide dismutase, $h s C R P$ high sensitivity $C$ reaction protein, ESR erythrocyte sedimentatio rate, IgA immunoglobulin $\mathrm{A}$, IgM immunoglobulin $\mathrm{M}$, IgG immunoglobulin G, C3 complement3, C4 complement4, $M$ mesangial hypercellularity, $E$ endocapillary hypercellularity, $S$ segmental glomerulosclerosis, $T$ tubular atrophy and interstitial fibrosis, $C$ crescents

${ }^{*} P<0.05,{ }^{*} P<0.001$

of IgAN patients had elevated Hcy levels and displayed more severe clinicopathologic characteristics, even in patients with normal renal function with eGFR $\geq 90 \mathrm{ml} /$ $\min / 1.73 \mathrm{~m}^{2}$. A longitudinal prospective study showed that elevated plasma Hcy levels may be a predictor of accelerated decline in renal function and future incidence of CKD [6]. An animal study implicated elevated
Hcy related to glomerular injury [26]. In both essential hypertension and general adult populations, high Hcy was associated with albuminuria independent of renal function [27, 28]. A prospective study showed that Hcy was an independent determinant of the development of albuminuria even after adjustment for eGFR [29]. In fact, Hcy is associated with vascular damage, endothelial 
dysfunction and cell proliferation, oxidative stress, lipid and lipoprotein metabolism and inflammation [30].

In addition, serum Hcy was positively correlated with hsCRP and ESR, but negatively correlated with SOD in the whole cohort of IgAN patients. Gori et al. revealed that interleukin- 6 and interleukin-1 were independent predictors of Hcy concentrations in elderly individuals [31]. Decreased SOD activity was observed in the kidneys of high Hcy rats [32]. SOD inhibits antioxidants and catalyzes superoxide conversion to $\mathrm{H}_{2} \mathrm{O}_{2}$. Hcy directly inhibits the activity of antioxidants, thereby disrupting SOD. On the other hand, elevated Hcy could induce oxidative stress, resulting in excessive superoxide production and SOD consumption [33]. The clinical results supported the findings of animal and cell model studies that Hcy promoted inflammation, redox imbalance and oxidative stress [34], which contributed to renal vascular damage. As a consequence, high Hcy levels may potentially induce renal injury and not only be the result of impaired renal function.

Of note, we investigated the relationship between Hcy and clinicopathologic characteristics in the whole cohort of 337 IgAN patients and then in the subgroup with eGFR $\geq 90 \mathrm{ml} / \mathrm{min} / 1.73 \mathrm{~m}^{2}$ to modify the effect of renal impairment. Our study showed consistent results that higher Hcy was associated with higher Scr, BUN, UA, ALB and $\mathrm{T}$ lesions but lower eGFR and HDL. However, there were opposite results in the correlation between Hcy and age, 24-h urine protein, and ESR at the early stage of 156 IgAN patients, which might be partially explained by the small sample size.

To date, whether Hcy is just a marker or plays a causal role in IgAN remains to be elucidated. The main strength of this study was that we assessed the association between serum Hcy and clinicopathologic characteristics in IgAN patients with normal renal function. We observed that elevated Hcy displayed more severe clinicopathologic characteristics, and pathologic $\mathrm{T}$ was an independent risk factor for elevated Hcy even in the early stage of IgAN. Our study prompted clinicians to pay more attention to Hcy levels in IgAN patients, especially at early stages. Previously, a randomized controlled trial in China suggested that Hcy-lowering therapy can significantly delay the progression of renal impairment among patients with mild-to-moderate CKD [35]. Further studies are needed in the future to discover the controversial relationship between kidney function and Hcy and confirm whether Hcy concentration intervention is beneficial to IgAN patients.

The present study had some limitations. First, because of the cross-sectional design of this study, we cannot obtain any causal inferences from the data. Second, as a single-center study, our study could not exclude the limits of races and regions, and its external validity may be limited. Third, the serum Hcy cutoff points differed among the previous studies $[4,11,36]$. However, Chinese expert consensus on hyperhomocysteinemia published in 2020 defined hyperhomocysteinemia as Hcy levels greater than $10 \mu \mathrm{mol} / \mathrm{L}$. A study of Hcy and Chinese patients with IgA nephropathy also defined hyperhomocysteinemia as Hcy levels greater than $10 \mu \mathrm{mol} / \mathrm{L}$ [11]. The population in our study was from China, so $10 \mu \mathrm{mol} / \mathrm{L}$ was selected as the Hcy cutoff in this study. Fourth, Hcy may be affected by folate, vitamin B12 and vitamin B6 status, which were not assessed in this study. In addition, there is a lack of follow-up data to assess the impact of baseline serum Hcy on renal and CVD outcomes in patients with IgAN.

\section{Conclusions}

IgAN patients with elevated serum Hcy displayed more severe clinicopathologic characteristics. Pathologic T was an independent risk factor associated with elevated Hcy in IgAN patients, especially at the early stage of IgAN.

\section{Abbreviations}

Hcy: Homocysteine; CVD: Cardiovascular disease; CKD: Chronic kidney disease; IgAN: IgA nephropathy; ESRD: End-stage renal disease; MR: Mendelian randomization; BP: Blood pressure; ANCA: Anti-neutrophil cytoplasm antibody; SBP: Systolic blood pressure; DBP: Diastolic blood pressure; MAP: Mean arterial pressure; HBP: Hypertension; Scr: Serum creatinine; eGFR: Estimated glomerular filtration rate; BUN: Blood urine nitrogen; UA: Uric acid; $\mathrm{Hb}$ : Hemoglobin; ALB: Serum albumin; Glu: Fasting glucose; CHOL: Cholesterol; TG: Triglyceride; LDL: Low-density lipoprotein-cholesterol; HDL: High-density lipoprotein-cholesterol; SOD: Superoxide dismutase; hsCRP: High sensitivity C reaction protein; ESR: Erythrocyte sedimentatio rate; IgA: Immunoglobulin A; IgM: Immunoglobulin M; IgG: Immunoglobulin G; C3: Complement3; C4: Complement4; CKD-EPI: Chronic Kidney Disease Epidemiology Collaboration; M: Mesangial hypercellularity; E: Endocapillary hypercellularity; S: Segmental glomerulosclerosis; T: Tubular atrophy and interstitial fibrosis; C: Crescents; CBS: Cystathione- $\beta$-synthase; CTL: $\gamma$-cystathionase; OR: Odds ratio; $95 \%$ Cl: 95\% Confidence interval.

\section{Supplementary Information}

The online version contains supplementary material available at https://doi. org/10.1186/s12882-021-02632-3.

Additional file 1: Supplement Table 1. Clinicopathologic characteristics comparison in the subgroup of IgAN patients with eGFR $\geq 90 \mathrm{ml} /$ $\mathrm{min} / 1.73 \mathrm{~m}^{2}$. Supplement Table 2. Correlations between Hcy and clinicopathologic parameters in the subgroup of IgAN patients with eGFR $\geq 90 \mathrm{ml} / \mathrm{min} / 1.73 \mathrm{~m}^{2}$. Supplement Table 3. Clinicopathologic features as factors of elevated Hcy by univariate and multivariate logistic regression analysis in whole populations with "Enter" method. Supplement Table 4. Clinicopathologic features as factors of elevated Hcy by univariate and multivariate logistic regression analysis in patients with eGFR $\geq 90 \mathrm{ml} / \mathrm{min} / 1.73 \mathrm{~m}^{2}$ with "Enter" method. 


\section{Acknowledgments}

We thank our colleagues at the Department of Nephrology, the Sun Yat-sen Memorial Hospital of Sun Yat-sen University, for their support and assistance during the study period.

\section{Authors' contributions}

LZZ and HQQ carried out data collection, interpretation of results and wrote the manuscript. YHB, LJ, WXN, HQY, XYC and LGX reviewed medical records. $\mathrm{LB}, \mathrm{LZZ}$ and $\mathrm{HQQ}$ performed data analysis. YQQ participated in the study design and coordination, and managing the research project. All authors have thoroughly reviewed the manuscript and approved the final manuscript for publication

\section{Funding}

This research was funded by financial from the National Natural Science Foundation of China (No. 81770709), the Guangzhou Municipal Science and Technology Project (No. 201904010142) and Sun Yat-sen Memorial Hospital Clinical Research and Cultivation Project (No. SYS-C-201903).

\section{Availability of data and materials}

The datasets used and/or analyzed during the current study available from the corresponding author on reasonable request.

\section{Declarations}

\section{Ethics approval and consent to participate}

All procedures performed in the study involving human participants were in accordance with the Declaration of Helsinki. This study was approved by the Medical Ethics Committee of Sun Yat-sen Memorial Hospital, Sun Yat-Sen University (approval number: No. SYSEC-KY-KS-2020-187). The informed consent was exempted by the Medical Ethics Committee of Sun Yat-sen Memorial Hospital, Sun Yat-Sen University.

\section{Consent for publication}

Not applicable.

\section{Competing interests}

The authors declare that they have no competing interests.

Received: 2 August 2021 Accepted: 7 December 2021

Published online: 23 February 2022

\section{References}

1. Kim J, Kim H, Roh H, Kwon Y. Causes of hyperhomocysteinemia and its pathological significance. Arch Pharm Res. 2018;41(4):372-83.

2. Chrysant S, Chrysant $\mathrm{G}$. The current status of homocysteine as a risk factor for cardiovascular disease: a mini review. Expert Rev Cardiovasc Ther. 2018;16(8):559-65.

3. Shih C, Shih Y, Chen J. The association between homocysteine levels and cardiovascular disease risk among middle-aged and elderly adults in Taiwan. BMC Cardiovasc Disord. 2021;21(1):191.

4. Cohen E, Margalit I, Shochat T, Goldberg E, Krause I. The relationship between the concentration of plasma homocysteine and chronic kidney disease: a cross sectional study of a large cohort. J Nephrol. 2019;32(5):783-9.

5. Perna A, Ingrosso D. Homocysteine and chronic kidney disease: an ongoing narrative. J Nephrol. 2019;32(5):673-5.

6. Levi A, Cohen E, Levi M, Goldberg E, Garty M, Krause I. Elevated serum homocysteine is a predictor of accelerated decline in renal function and chronic kidney disease: a historical prospective study. Eur J Intern Med. 2014;25(10):951-5.

7. Kong X, Ma X, Zhang C, Su H, Xu D. Hyperhomocysteinemia increases the risk of chronic kidney disease in a Chinese middle-aged and elderly population-based cohort. Int Urol Nephrol. 2017:49(4):661-7.

8. Wyatt RJ, Julian BA. IgA nephropathy. N Engl J Med. 2013;368(25):2402-14

9. Le W, Liang S, Hu Y, Deng K, Bao H, Zeng C, et al. Long-term renal survival and related risk factors in patients with IgA nephropathy: results from a cohort of 1155 cases in a Chinese adult population. Nephrol Dial Transplant. 2012;27(4):1479-85.

10. Duan S, Liu S, Sun X, Zheng Y, Liu L, Yao F, et al. Potential association of hyperhomocysteinemia with the progression of IgA nephropathy: a retrospective study. Chin Med J. 2014;127(10):1849-52.

11. Zhang YM, Zhou XJ, Shi SF, Liu LJ, Lyu JC, Zhang H. Homocysteine and IgA nephropathy: observational and Mendelian randomization analyses. Chin Med J. 2020;133(3):277-84.

12. Stevens LA, Claybon MA, Schmid CH, Chen J, Horio M, Imai E, et al. Evaluation of the chronic kidney disease epidemiology collaboration equation for estimating the glomerular filtration rate in multiple ethnicities. Kidney Int. 2011;79(5):555-62.

13. Trimarchi H, Barratt J, Cattran D, Cook H, Coppo R, Haas M, et al. Oxford classification of IgA nephropathy 2016: an update from the IgA nephropathy classification working group. Kidney Int. 2017;91(5):1014-21.

14. Ostrakhovitch EA, Tabibzadeh S. Homocysteine and age-associated disorders. Ageing Res Rev. 2019;49:144-64.

15. Katsiki N, Mikhailidis D. Emerging vascular risk factors in women: any differences from men? Curr Med Chem. 2015;22(31):3565-79.

16. Cohen E, Margalit I, Shochat T, Goldberg E, Krause I. Gender differences in homocysteine concentrations, a population-based cross-sectional study. Nutr Metab Cardiovasc Dis. 2019;29(1):9-14.

17. Sobczak AJ. The effects of tobacco smoke on the homocysteine level--a risk factor of atherosclerosis. Addict Biol. 2003:8(2):147-58.

18. Dimitrova KR, DeGroot K, Myers AK, Kim YD. Estrogen and homocysteine. Cardiovasc Res. 2002;53(3):577-88.

19. Ye Z, Zhang Q, Li Y, Wang C, Zhang J, Ma X, et al. High prevalence of hyperhomocysteinemia and its association with target organ damage in Chinese patients with chronic kidney disease. Nutrients. 2016;8(10):645.

20. Togawa T, Sengupta S, Chen H, Robinson K, Nonevski I, Majors A, et al. Mechanisms for the formation of protein-bound homocysteine in human plasma. Biochem Biophys Res Commun. 2000;277(3):668-74.

21. Amin $\mathrm{H}$, El-Sayed $\mathrm{M}$, Leheta $\mathrm{O}$. Homocysteine as a predictive biomarker in early diagnosis of renal failure susceptibility and prognostic diagnosis for end stages renal disease. Ren Fail. 2016;38(8):1267-75.

22. Long Y, Nie J. Homocysteine in renal injury. Kidney Dis. 2016;2(2):80-7.

23. Friedman AN, Bostom AG, Selhub J, Levey AS, Rosenberg $I H$. The kidney and homocysteine metabolism. J Am Soc Nephrol. 2001;12(10):2181-9.

24. House JD, Brosnan ME, Brosnan JT. Characterization of homocysteine metabolism in the rat kidney. Biochem J. 1997;328(Pt 1):287-92.

25. Li N, Chen L, Muh RW, Li P-L. Hyperhomocysteinemia associated with decreased renal transsulfuration activity in Dahl $\mathrm{S}$ rats. Hypertension. 2006;47(6):1094-100

26. Cao L, Lou X, Zou Z, Mou N, Wu W, Huang X, et al. Folic acid attenuates hyperhomocysteinemia-induced glomerular damage in rats. Microvasc Res. 2013;89:146-52.

27. Marti F, Vollenweider P, Marques-Vidal P-M, Mooser V, Waeber G, Paccaud $F$, et al. Hyperhomocysteinemia is independently associated with albuminuria in the population-based CoLaus study. BMC Public Health. 2011;11:733.

28. Kuang Z, Wang $Y$, Feng $S$, Jiang $L$, Cheng W. Association between plasma homocysteine and microalbuminuria in untreated patients with essential hypertension: a case-control study. Kidney Blood Press Res. 2017:42(6):1303-11.

29. Jager A, Kostense PJ, Nijpels G, Dekker JM, Heine RJ, Bouter LM, et al. Serum homocysteine levels are associated with the development of (micro)albuminuria: the Hoorn study. Arterioscler Thromb Vasc Biol. 2001;21(1):74-81.

30. Ganguly P, Alam S. Role of homocysteine in the development of cardiovascular disease. Nutr J. 2015;14:6

31. Gori AM, Corsi AM, Fedi S, Gazzini A, Sofi F, Bartali B, et al. A proinflammatory state is associated with hyperhomocysteinemia in the elderly. Am J Clin Nutr. 2005;82(2):335-41.

32. Hwang S-Y, Siow YL, Au-Yeung KKW, House J, Karmin O. Folic acid supplementation inhibits NADPH oxidase-mediated superoxide anion production in the kidney. Am J Physiol Renal Physiol. 2011;300(1):F189-98.

33. Fu $Y$, Wang $X$, Kong W. Hyperhomocysteinaemia and vascular injury: advances in mechanisms and drug targets. Br J Pharmacol. 2018;175(8):1173-89.

34. Ostrakhovitch E, Tabibzadeh S. Homocysteine in chronic kidney disease. Adv Clin Chem. 2015:72:77-106. 
35. Xu X, Qin X, Li Y, Sun D, Wang J, Liang M, et al. Efficacy of folic acid therapy on the progression of chronic kidney disease: the renal substudy of the China stroke primary prevention trial. JAMA Intern Med. 2016;176(10):1443-50.

36. Tak Y, Jeong D, Kim Y, Lee S, Lee J, Song S, et al. Hyperhomocysteinaemia as a potential marker of early renal function decline in middleaged Asian people without chronic kidney disease. Int Urol Nephrol. 2016;48(2):239-48.

\section{Publisher's Note}

Springer Nature remains neutral with regard to jurisdictional claims in published maps and institutional affiliations.

- fast, convenient online submission

- thorough peer review by experienced researchers in your field

- rapid publication on acceptance

- support for research data, including large and complex data types

- gold Open Access which fosters wider collaboration and increased citations

- maximum visibility for your research: over $100 \mathrm{M}$ website views per year

At BMC, research is always in progress.

Learn more biomedcentral.com/submissions 